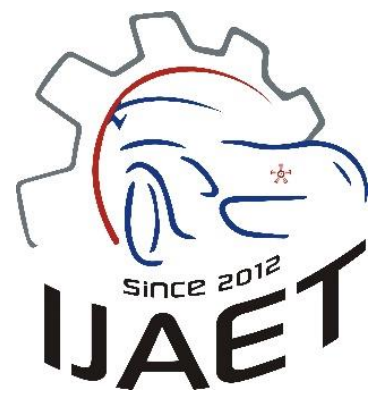

e-ISSN: 2146 - 9067

International Journal of Automotive

Engineering and Technologies

journal homepage: http://ijaet.academicpaper.org

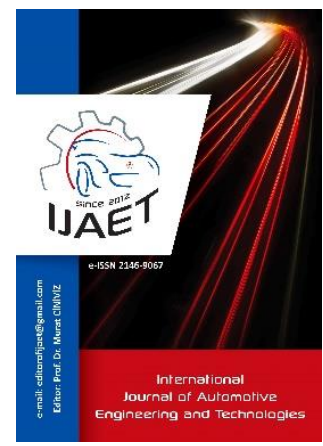

Original Research Article

\title{
Determining optimal artificial neural network training method in predicting the performance and emission parameters of a biodiesel-fueled diesel generator
}

\author{
Şehmus Altun ${ }^{1}$, Ömer Faruk Ertuğrul ${ }^{2 *}$ \\ ${ }^{1}$ Batman University, Faculty of Technology, Department of Automotive Engineering, Turkey \\ ${ }^{2}$ Batman University, Faculty of Engineering-Architecture, Department of Electrical-Electronic Engineering, Turkey
}

\author{
ARTICLE INFO \\ * Corresponding author \\ omerfarukertugrul@gmail.com \\ Received: June 10, 2017 \\ Accepted: January 08, 2018 \\ Published by Editorial Board \\ Members of IJAET \\ (C) This article is distributed by \\ Turk Journal Park System \\ under the CC 4.0 terms and \\ conditions.
}

\begin{abstract}
Artificial neural network (ANN) methods were employed and suggested in modeling the emissions and performance of a diesel generator fueled with waste cooking oil derived biodiesel during steady-state operation. These papers are generally built on determining optimal network structure, but the modelling accuracy of an ANN is also highly dependent on employed training method. In modeling, operating conditions and fuel blend ratio were used as the inputs while the performance and emission parameters were the outputs. The modeling results obtained by conventional ANNs that were trained by back propagation (BP) learning algorithm, radial basis function (RBF), and extreme learning machine (ELM) were compared with experimental results and each other. The accuracy of the estimations by ELM was above $95 \%$ for all the output parameters except for specific fuel consumption and thermal efficiency. Moreover, ELM performed better than BP and RBF with lower mean relative error (MRE) in case where the emissions were estimated. The ELM provided correlation coefficients of 0.987, 0.950 and 0.996 for unburned hydrocarbons ( $\mathrm{HCs}$ ), nitrogen oxides $\left(\mathrm{NO}_{\mathrm{x}}\right)$ and smoke opacity (SO), respectively, while for BP, they were $0.973,0.818,0.993$, and for RBF, 0.975, 0.640 and 0.981 . The most suitable training function for each emission and performance parameters of diesel generator was determined based on obtained accuracies.
\end{abstract}

Keywords: Extreme learning machine; Artificial Neural Network; Radial Basis Function; Back propagation; Biodiesel; Diesel generator; Emissions

\section{Introduction}

The increasing concerns on global warming and environmental pollution caused by excessive use of fossil fuels, which have limited reserves, have driven the researches to investigate alternative fuels and their efficient use. Biodiesel, which is obtained from oil and fats via transesterification, is considered as diesel fuel surrogate thanks to its emission-reducing potential, its renewability, etc. [1-3]. However, its high nitrogen oxides $\left(\mathrm{NO}_{\mathrm{x}}\right)$ emissions and production costs are still barriers to its wide use [4]. On the other hand, the use of biofuels is endorsed by the European Commission in the 2009/28/CE directive, and the anticipated target is now to reach $10 \%$ of biofuel use by the end of 2020 [5]. Therefore, special devotion has been given to biodiesel use and obliged to use in specified ratios and biodiesel fuels have been reported to reduce diesel emissions except for 
$\mathrm{NO}_{\mathrm{x}}$ emissions [6-9]. However, for dieselpowered generators run by biodiesel, no consensus has been reported on the changes in emissions likely thanks to the lack of works [1011]. Numerous experiments should be completed to elucidate the change in emissions of this type of engines, but testing the engine with various fuel-blends under a wide range of working conditions are time-consuming and costly, and this is not an eco-friendly approach. Alternatively, the estimations can be made by machine learning methods, which can estimate the outputs of unknown situations by modeling the physical phenomena in complex systems using experimental results with significantly less engineering effort and high performance [12].

Artificial neural network (ANN), which is one of the most popular machine learning method, known as "parallel distributed processing" or "connectionist model" is based on the findings of biological neurons [12]. Its theory depends on the studies of McCulloch and Pitts in 1943 [13], and it can be expressed as a simple and small sized neuron network that has learning, remembering and deciding with generalization of previous knowledge. ANN requires training for determining optimal weights and biases therefore before modeling a system there must a dataset to describe the system due to its measured inputs and outputs. Recently, ANN techniques have often been used for modeling of biofuel-fueled engines. For instance, Yap and Karri [14] demonstrated the series ANN model as generic virtual power and emission sensors. Kökkülünk et al. [15] estimated the emissions with a very high accuracy by means of the designed ANN structures. Kumar et al. [16] and Patel and Patel [17] developed an ANN model based on a standard back propagation (BP) algorithm to predict the engine responses while Kumar et al. [18] investigated the applicability of radial basis function neural network (RBF), which is a relatively new class of ANNs, and Shi et al. [19] employed RBF for building model of marine diesel-generator. Additionally, Wong et al. employed extreme learning machine (ELM), which was recently introduced $[20,21]$, in the modeling of biodiesel-engine performance [22, 23].

Hence, it is preferred to build a model to estimate the diesel-powered generator run by biodiesel and diesel-biodiesel blends responses. According to the authors' best knowledge, an extensive study has not been determining the optimal training method to this type of system, yet. Therefore, waste cooking (sunflower) oil derived biodiesel, ultra-low sulphur diesel and their blends ( $20 \%$ and $50 \%$ in vol.) were tested in a 3-cylinder diesel engine-powered generator under constant speed and variable load conditions to obtain training data, which were then used to build a model. Afterwards, ANNs, were optimized by determining transfer functions and number of neurons in the hidden layer, and finally, the performance of ELM, BP and RBF based on estimated engine responses, were compared with each other in terms of their accuracy and mean relative error.

In the rest of paper, material and experimental procedure followed for obtaining the training data were explained in Section 2, while the structures of ANN and its learning methods were briefly presented in Section 3. The optimization of the parameters was also studied in Section 4. The modelling results and the comparison of the performance of applied methods were discussed in Section 5. Finally, the concluding notes were presented in Section 6.

\section{Material and experimental procedure}

The employed dataset was generated from the biodiesel, which was manufactured from transesterification of waste cooking (sunflower) oil that was collected from University cafeteria, with methanol in the presence of an alkali catalyst to use in engine tests. Detailed information about this production processes can be found in Ref. [24]. The reference fuel was an ultra-low Sulphur diesel fuel (ULSD), supplied by local fueling station. Density and kinematic viscosity of biodiesel was found as $884.7 \mathrm{~kg} / \mathrm{m}^{3}$ and $4.7 \mathrm{~mm}^{2} / \mathrm{s}$, respectively, which are higher than those of ULSD, $835 \mathrm{~kg} / \mathrm{m}^{3}$ and $2.8 \mathrm{~mm}^{2} / \mathrm{s}$. The energy content of biodiesel was lower than that of ULSD by about $11 \%$. Tests were carried out in a 3-cylinder and direct-injection diesel engine-powered generator set. It is a type of GEN-SET that represents a large population for energy generation in institutional facilities. Fuel consumption was measured by using an electronic scale and digital chronometer. Emissions were recorded by Capelec Cap 3200 
analyzer. Fuels were tested at a constant engine speed of $1500 \mathrm{rpm}$ and at different loads. Each test was repeated three times and the average values were used. Specific-fuel consumption (SFC) and efficiency were calculated by the following equations.

$S F C=\frac{m_{f u e l} \times 3600}{P e}(g / k W h)$

Efficiency $=\frac{3.6 \times 10^{6}}{S F C \times H u}(\%)$

where $m_{\text {fuel }}$ is the mass flow rate of the fuel as $\mathrm{g} / \mathrm{s}, P e$ is the power output in $\mathrm{kW}, \mathrm{Hu}$ is the lower heating value of the fuels in $\mathrm{kJ} / \mathrm{kg}$. Only 28 samples for each parameter corresponding to a constant speed, different loads and fuel blends were collected from the experiments [24]. From the viewpoint of machine learning (ML) approaches, 28 points are reflected as a small dataset that leads to the problem of data scarcity. This reduces the accuracy of ML since training is stopped when the error does not decrease anymore or when the error reached to the desired error value. On the other hand, it is reported that the increase in the amount of training data causes a decrease in the generalization error [12]. The model performance depends on the representational power of the dataset and also the number of hidden units (neuron and layer) with the activation function [25]. The change in all the parameters is proportional to the load of the engine and biodiesel content in the fuel, except for smoke opacity (SO) whose values at lowloads were recorded as $0 \%$ for blends, leading to decrease the accuracy as " 0 " values do not give enough knowledge about the characteristics of the system.

\section{Modeling of the biodiesel-fueled generator}

Artificial neural network (ANN), which is based on the human neural system, have been successfully employed in many purposes [25, 26]. In ANN, many neurons are connected with each other and an ANN is formed based on these connections. ANNs are trained by experiences (training set) and in the training stage, weights and biases are optimized by minimizing training errors. The output of ANN can be calculated by Eq. (3) [27].

$y=\sum_{j=1}^{m} \beta_{j, k} g\left(\sum_{i=1}^{n} w_{i, j} x_{i}+b_{j}\right)$
Here, $x_{i}, y, w_{i, j}, \beta_{j, k}, b_{j}$, and $g($.$) represent$ input, output, weights in the input neurons, weights of output neurons, biases of the neurons in hidden layer, and the activation function, respectively. Furthermore, $\mathrm{n}, \mathrm{m}$, and $\mathrm{k}$ show number of neurons in the input, hidden, and output layers, respectively [12].

ELM- ELM is a kind of learning methods in ANN that has a number of attractive features such as an extremely fast train stage and a high generalization performance [28] and train speed on account of the fact that the output weights can be analytically determined by Moore-Penrose generalized inverse method when the input weights, hidden layer and its biases are randomly assigned. But it has two limitations, which are the employed transfer function is infinitely differentiable and also, the number of neurons in the hidden layer is less than the number of samples in the training dataset. ELM network architecture is shown in Figure 1.

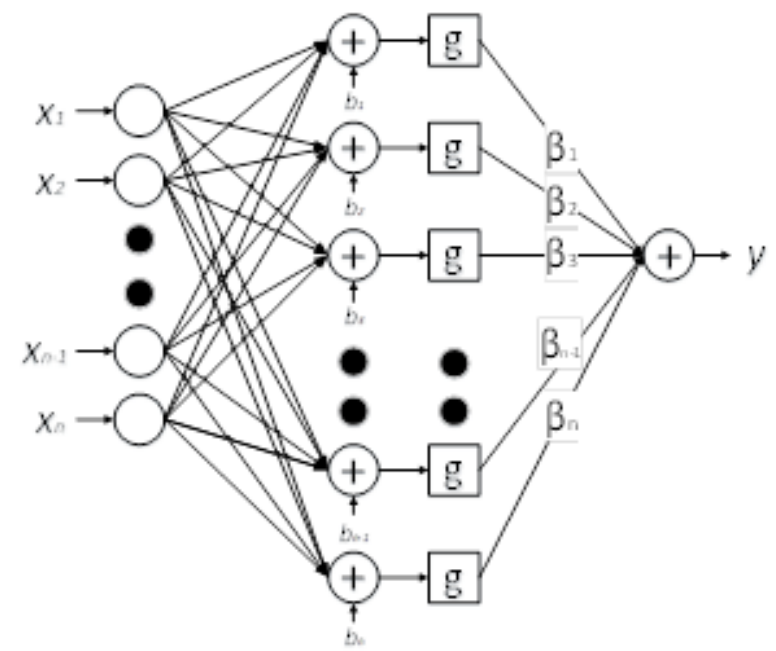

Figure 1. A single hidden layer neural network

The outputs can be calculated by using Eq. (4) $[20,21]$ and can be rewritten as:

$H \beta=y$

where $H$ is hidden layer output matrix stands for Eq. (5) [20, 21]:

$H\left(w_{i, j}, b_{j}, x_{i}\right)=$
$\left[\begin{array}{ccc}g\left(w_{1,1} x_{1}+b_{1}\right) & \cdots & g\left(w_{1, m} x_{m}+b_{m}\right) \\ \vdots & \ddots & \vdots \\ g\left(w_{n, 1} x_{n}+b_{1}\right) & \cdots & g\left(w_{n, m} x_{m}+b_{m}\right)\end{array}\right]$

Finally, the weights of neurons in the output layers $\left(\beta_{1 \cdots m, 1 \cdots k}\right)$ is analytically computed by 
Moore-Penrose generalized inverse method (Eq. (4)).

$\hat{\beta}=H^{+} y$

where $H^{+}$denotes the Moore-Penrose generalized inverse matrix of $\mathrm{H}$.

BP- BP, a gradient descent learning algorithm, is a conventional ANN method that has emerged as the standard algorithm for the training of multilayer perceptron's against which other learning algorithms are often benchmarked [24]. In BP, optimum weights are determined by back propagating the errors from output layer [25, 29]. As a simple way, the errors are taken into account in backward direction. Although backpropagation has been successfully employed in many research areas, it has some major problems such that its long training stage and the probability of falling into local minima [29]. In delta rule the weights are changed by;

$\Delta w_{j k}=-\gamma \frac{\partial E}{\partial w_{j k}}$

where $\gamma$ is constant of proportionality and $E$ is the error term (mean square error) which is found by Eq. (8).

$E=\frac{1}{2} \sum_{i=1}^{N}\left(d_{i}-y_{i}\right)^{2}$

where $\mathrm{N}$ is the number of data at train dataset, $\mathrm{d}$ is the desired output, $\mathrm{y}$ is actual output and $i$ is the data order. The error of train dataset was back propagated to optimize network parameters.

By chain rule $\frac{\partial E}{\partial w_{j k}}=\frac{\partial E}{\partial G} \frac{\partial G}{\partial w_{j k}}$ while $y=\frac{\partial G}{\partial w_{j k}}$ and $\delta_{k}=-\frac{\partial E}{\partial G_{k}}$ can be defined where $\mathrm{G}$ is defined as the sum of all inputs entering the neuron. Then the weight update rule for each neuron can be calculated by Eq. (9).

$\Delta w_{j, k}=\gamma \delta_{k} y_{j}$

Here in $\delta_{\mathrm{k}}$ must be computed for each unit $\mathrm{k}$ in the network. $\delta_{\mathrm{k}}$ for output layer $\delta_{o}=(d-$ y) $\frac{\partial y_{o}}{\partial G_{o}}$ and for the hidden layer $\delta_{h}=$ $\frac{\partial y_{o}}{\partial G_{o}} \sum_{o=1}^{N_{o}} \delta_{o} w_{h o}$ where $\mathrm{N}_{\mathrm{o}}$ is the number of neurons in hidden layer, while $G_{0}$ shows the value that come to the output neuron [25].

RBF- RBF has been offered by Broomhead and Lowe [30] and it has the following differences from BP: there is not any assigned weight in the input layer and the transfer function of hidden layer is radial basis function as shown in Eq. (10).

$f(x)=\exp \left[-(x-M)^{2} / 2 \sigma^{2}\right]$

where $M$ and $\sigma$ are mean and standard deviation respectively. In RBF, the linear mapping from hidden layer to output layer is the single parameter that is adjusted in the learning process therefore it does not suffer from local minima.

These three models were employed for modeling the performance and emission parameters of a biodiesel-fueled generator. The data obtained at constant engine speed and different loads were used as inputs while SFC, thermal efficiency (TE), exhaust gas temperature (EGT), hydrocarbons ( $\mathrm{HCs}), \mathrm{NO}_{\mathrm{x}}$ and smoke opacity (SO) were outputs. In this study, ELM was used as the base model while $\mathrm{BP}$ and RBF were for testing the performance of ELM and to determine the most suitable learning model for each parameter of biodieselfueled generator.

\section{Optimizing the parameters}

Determining the structure and learning coefficients is the most important stage in developing model, and this may increase the success. These coefficients are related to the characteristics of the dataset. Therefore, to increase the success that obtained from each model, for each dataset, the parameters of learning algorithms were determined by trials. Besides, to make a more equitable comparison among the models, the parameters of all methods used should be optimized for each parameter. To obtain optimum network parameters, many iterative learning steps may be required in order to obtain a good learning performance [21]. In ELM, the number of hidden layer and neurons is limited to single hidden layer and the number of observations, respectively [31]. Therefore, the networks were tested with 5-25 neurons in one hidden layer. Also in BP, hard-limit (hardlim), log-sigmoid (logsig), hyperbolic tangent sigmoid (tansig), triangular basis (tribas) and radial basis (radbas) transfer functions were used in trials since in theory, any activation function that has a first derivative can be used in BP. Unlike BP, ELM can use any nonlinear activation function. In this study sigmoid (sig), sinus (sin), hardlim, tribas 
and radbas transfer functions were tested for ELM. In RBF, the optimization was done in terms of the spread of radial basis function. For having a fair comparison, the general structure of ANN was picked out to be a single hidden layer feed forward neural network (SLFN). Here, the input layer consists of three neurons which represent the speed and load and fuel blending ratio. The number of neurons in hidden layer and transfer functions was determined after an optimizing stage which will be described in this section. Finally, one output neuron is used as unique network structures were determined for each parameter. In Figure 2, a flow chart that shows the optimization stage of data.

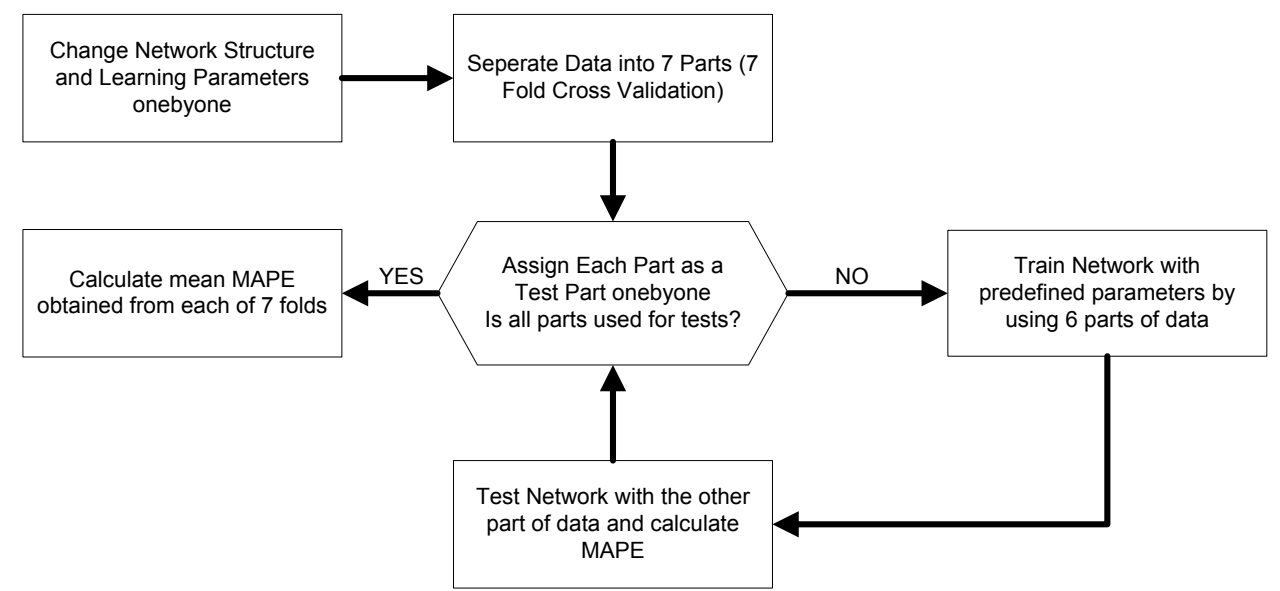

Figure 2. Flow chart of optimization stage

The optimal values and transfer functions were obtained by picking the network parameters, and it was shown by the least average mean absolute percentage error (MAPE), which was calculated following equation (MAPE = $\left.\frac{100 \%}{n} \sum_{i=1}^{n}\left|\frac{f_{i}-y_{i}}{y_{i}}\right|\right)$, where $f$ is estimated and $y$ is true value. In 7-fold cross validation, the data is partitioned into 7 equal sized (each subset contains 4 observations) subsets and one is used as a validation (test) data and the rest is used as training data [32]. The optimized parameters, which are transfer function (TF), number of neurons in the hidden layer (NN) and spread, are sorted in Table 1.

Table 1. Optimized network parameters for models employed

\begin{tabular}{lccccc}
\hline \multirow{2}{*}{ Parameters } & \multicolumn{2}{c}{ ELM } & \multicolumn{2}{c}{ BP } & RBF \\
\cline { 2 - 6 } & TF & NN & TF & NN & Spread \\
\hline BSFC & Sin & 25 & tansig & 10 & 1.9 \\
BTE & Sin & 20 & logsig & 5 & 1.0 \\
EGT & Sin & 25 & tansig & 5 & 1.1 \\
HC & Sin & 20 & logsig & 5 & 1.1 \\
NO $_{x}$ & Sin & 20 & logsig & 5 & 3.2 \\
SO & Tribas & 15 & tansig & 5 & 7.8 \\
\hline
\end{tabular}

\section{Results and discussion}

For minimizing the classification errors that arise from data distribution, the estimations were done by leave-one-out cross-validation [32] where a single data in the dataset are used as a validation data, and the rest is used as the training data. The average of all estimation accuracies of optimized ELM, BP and RBN learning algorithms for each engine parameter are assumed as the accuracy of ML systems. The relative errors (RE) calculated for the estimations of the engine fueled with pure biodiesel (B100) as example, which are found by using following equation $\left(R E=\left|\frac{f-y}{y}\right|\right)$, are tabulated in Tables 2 and 3, and mean relative errors (MRE) are given in Table 4. As can be seen in Tables, amongst models employed, ELM is a useful tool for estimating $\mathrm{NO}_{\mathrm{x}}$, smoke and EGT while BP is more successful in estimating of SFC and $\mathrm{HC}$ in relation to RE and 
MRE. For TE, it is seen that ELM and BP have very similar performance. In case of RBF, the values of mean relative error are above in all cases.

Table 2. The Relative Errors of the performance parameters for biodiesel

\begin{tabular}{ccccccccc}
\hline Estimated & Power & Measured & Estimated & \multicolumn{2}{c}{ Relative } & Estimated & \multicolumn{2}{c}{ Relative } \\
Parameters & Output & Value & by ELM & Error & by BP & Error & by RBF & $\begin{array}{c}\text { Relative } \\
\text { Error }\end{array}$ \\
\hline & 1.4 & 947.14 & 996.93 & 0.0526 & 964.6 & 0.0184 & 984.33 & 0.0393 \\
& 2.9 & 591.72 & 592.04 & 0.0005 & 565.69 & 0.0440 & 675.49 & 0.1416 \\
SFC & 4 & 489 & 446.32 & 0.0873 & 439.86 & 0.1005 & 456.63 & 0.0662 \\
$(\mathrm{~g} / \mathrm{kWh})$ & 5.3 & 417.74 & 422.82 & 0.0122 & 435.59 & 0.0427 & 423.28 & 0.0133 \\
& 5.8 & 411.72 & 417.39 & 0.0138 & 421.9 & 0.0247 & 406.17 & 0.0135 \\
& 6.5 & 423.69 & 415.57 & 0.0192 & 411.45 & 0.0289 & 431.57 & 0.0186 \\
& 7 & 420 & 498.94 & 0.1880 & 403.72 & 0.0388 & 396.52 & 0.0559 \\
\hline & 1.4 & 10.14 & 10.41 & 0.0266 & 9.94 & 0.0197 & 13.39 & 0.3205 \\
& 2.9 & 16.22 & 16.42 & 0.0123 & 17.42 & 0.0740 & 18.39 & 0.1338 \\
& 4 & 19.63 & 19.06 & 0.0290 & 20.54 & 0.0464 & 23.04 & 0.1737 \\
TE $(\%)$ & 5.3 & 22.98 & 23.23 & 0.0109 & 21.9 & 0.0470 & 22.76 & 0.0096 \\
& 5.8 & 23.32 & 23.14 & 0.0077 & 22.04 & 0.0549 & 22.71 & 0.0262 \\
& 6.5 & 22.66 & 23.38 & 0.0318 & 23.65 & 0.0437 & 23.02 & 0.0159 \\
& 7 & 22.86 & 23.63 & 0.0337 & 23.12 & 0.0114 & 22.67 & 0.0083 \\
\hline & 1.4 & 136 & 120.43 & 0.1145 & 142.14 & 0.0452 & 154.62 & 0.1369 \\
& 2.9 & 150 & 162.28 & 0.0819 & 165.77 & 0.1051 & 164.35 & 0.0957 \\
& 4 & 166 & 171.48 & 0.0330 & 171.61 & 0.0338 & 174.59 & 0.0518 \\
EGT $\left({ }^{\circ} \mathrm{C}\right)$ & 5.3 & 176 & 172.39 & 0.0205 & 181.34 & 0.0303 & 178.63 & 0.0149 \\
& 5.8 & 205 & 203.43 & 0.0077 & 201.02 & 0.0194 & 202.92 & 0.0101 \\
& 6.5 & 229 & 228.25 & 0.0033 & 227.74 & 0.0055 & 234.34 & 0.0233 \\
& 7 & 274 & 280.1 & 0.0223 & 280.58 & 0.0240 & 278.94 & 0.0180 \\
\hline
\end{tabular}

Table 3. The Relative Errors of the emission parameters for biodiesel

\begin{tabular}{ccccccccc}
\hline Estimated & Power & Measured & Estimated & Relative & Estimated & \multicolumn{2}{c}{ Relative } & Estimated \\
Parameters & Output & Value & by ELM & Error & by BP & Error & by RBF & Error \\
\hline & 1.4 & 27 & 29.73 & 0.1011 & 27.23 & 0.0085 & 24.92 & 0.0770 \\
& 2.9 & 51 & 52.03 & 0.0202 & 48.23 & 0.0543 & 61.66 & 0.2090 \\
& 4 & 77 & 71.64 & 0.0700 & 75.03 & 0.0256 & 76.15 & 0.0110 \\
$\mathrm{HC}(\mathrm{ppm})$ & 5.3 & 99 & 106.81 & 0.0789 & 115.03 & 0.1619 & 95.27 & 0.0377 \\
& 5.8 & 116 & 111.15 & 0.0418 & 104.71 & 0.0973 & 103.64 & 0.1066 \\
& 6.5 & 129 & 124.38 & 0.0358 & 121.46 & 0.0585 & 122.47 & 0.0506 \\
& 7 & 132 & 138.61 & 0.0501 & 135.67 & 0.0278 & 131.25 & 0.0057 \\
\hline & 1.4 & 371 & 339.03 & 0.0862 & 416.39 & 0.1224 & 492.89 & 0.3285 \\
& 2.9 & 565 & 614.04 & 0.0868 & 649.01 & 0.1487 & 697.15 & 0.2339 \\
& 4 & 707 & 632.15 & 0.1059 & 684.16 & 0.0323 & 691.76 & 0.0216 \\
NO $_{\mathrm{x}}(\mathrm{ppm})$ & 5.3 & 853 & 910.25 & 0.0671 & 813.82 & 0.0460 & 975.1 & 0.1431 \\
& 5.8 & 960 & 1000.79 & 0.0425 & 881.29 & 0.0820 & 927.16 & 0.0342 \\
& 6.5 & 973 & 996.92 & 0.0246 & 923.53 & 0.0508 & 1005.83 & 0.0337 \\
& 7 & 986 & 956.83 & 0.0296 & 901.14 & 0.0861 & 862.04 & 0.1257 \\
\hline & 1.4 & 0 & 0 & 0 & 0 & 0 & 0 & 0 \\
& 2.9 & 0 & 0 & 0 & 0 & 0 & 0 & 0 \\
& 4 & 0 & 0.01 & 0 & 0 & 0 & 0 & 0 \\
$\mathrm{SO}(\%)$ & 5.3 & 7.4 & 7.2 & 0.0270 & 8.43 & 0.1392 & 8.05 & 0.0878 \\
& 5.8 & 17.2 & 17.72 & 0.0302 & 20.49 & 0.1913 & 19.43 & 0.1300 \\
& 6.5 & 28.7 & 28.69 & 0.0003 & 29.86 & 0.0404 & 28.76 & 0.0021 \\
& 7 & 32.3 & 32.06 & 0.0074 & 32.33 & 0.0009 & 32.4 & 0.0031 \\
\hline
\end{tabular}


Table 4. Mean Relative Errors

\begin{tabular}{lcccccc}
\hline Method & SFC & TE & EGT & HC & NO $_{x}$ & SO \\
\hline ELM & 0.0559 & 0.0470 & 0.0357 & 0.0457 & 0.0608 & 0.0520 \\
BP & 0.0324 & 0.0430 & 0.0729 & 0.0525 & 0.1170 & 0.0724 \\
RBF & 0.0367 & 0.1325 & 0.0709 & 0.0605 & 0.1912 & 0.0935 \\
\hline
\end{tabular}

The comparisons of estimated and measured results, which can be used to illustrate the deviations from the actual value for estimations, for all parameters of using biodiesel are shown in Figure 3. As shown in the figure, the actual and estimated values are very close to each other, however, $\mathrm{HC}$ and $\mathrm{NO}_{\mathrm{x}}$ values do not
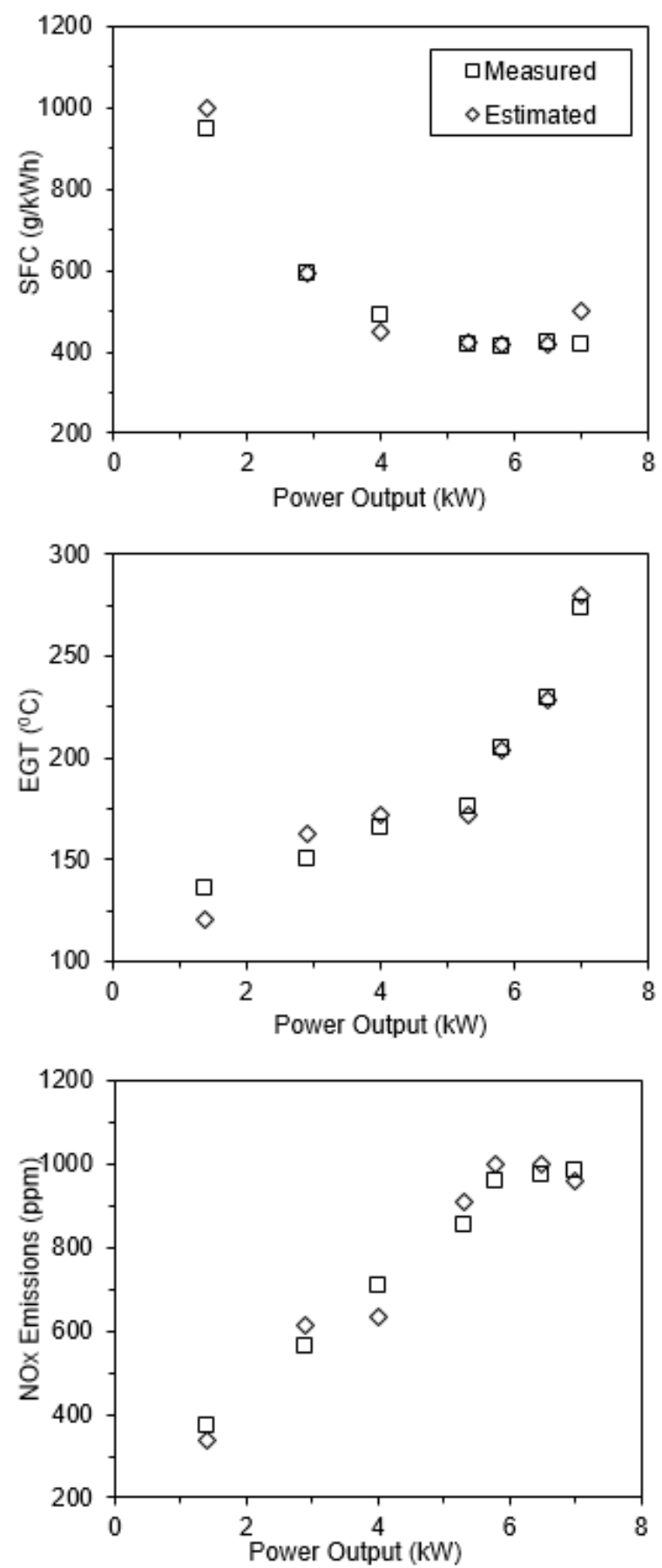

match enough especially at high loads. This is mainly due to the nature of emissions that it is hard to estimate due to the difficulty of the burning process. Furthermore, there was an interesting trend for estimating SO values; they were almost exactly fit with the experimental values.
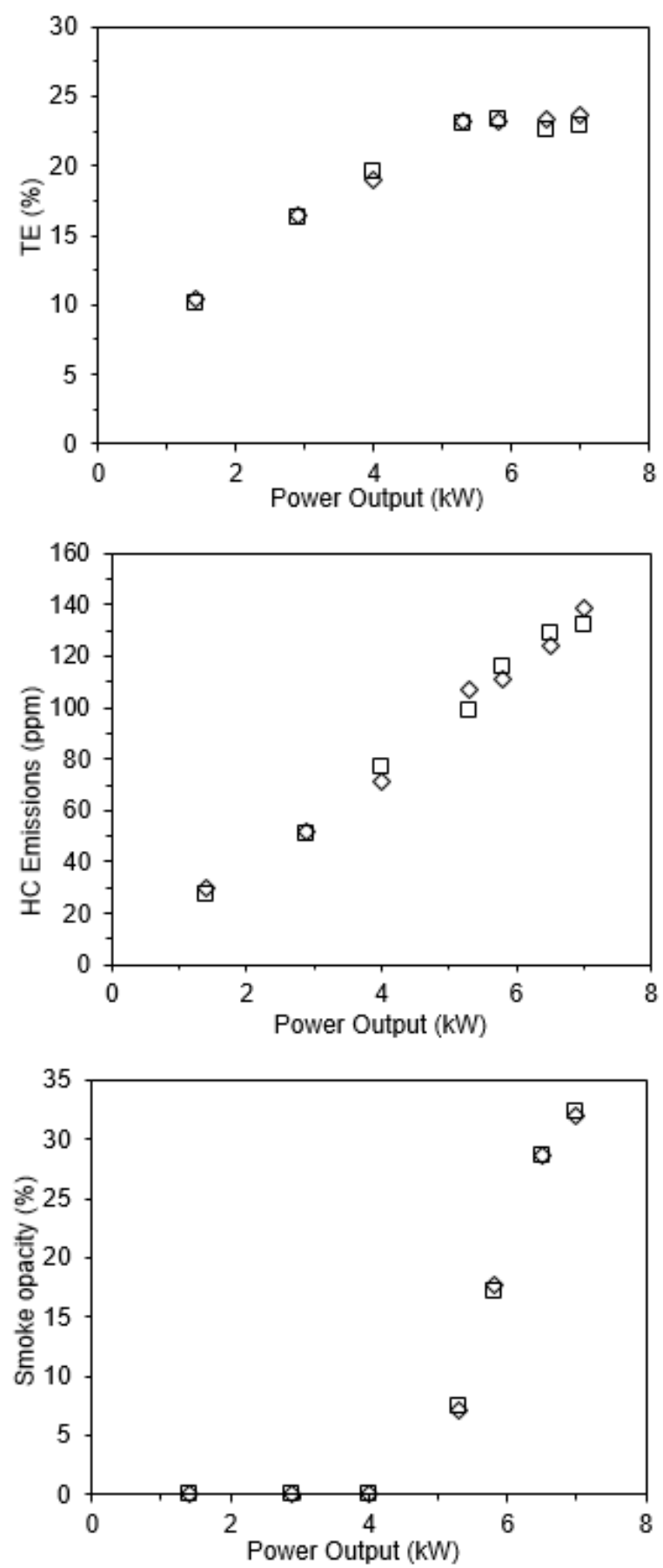

Figure 3. Comparison the measured and estimated parameters obtained for B100 
To compare the performance of models, the estimation error was presented by MAE, RMSE, MAPE, $\mathrm{R}^{2}$ in Table 5. They are evaluated against the experimental data using following equations; mean absolute error $(M A E=$ $\left.\frac{1}{N} \sum_{i=1}^{n}\left|f_{i}-y_{i}\right|\right), \quad$ root mean square error $\left(R M S E=\sqrt{E\left[(f-y)^{2}\right]}\right)$, mean absolute percentage error $\left(M A P E=\frac{100 \%}{n} \sum_{i=1}^{n}\left|\frac{f_{i}-y_{i}}{y_{i}}\right|\right)$ and coefficient of determination $\left(R^{2}=1-\right.$ $\left.\frac{\sum_{i=1}^{n}\left(f_{i}-y_{i}\right)^{2}}{\sum_{i=1}^{n} y_{i}^{2}}\right)$, where $E$ is expected, $f$ is estimated and $y$ is true value.

Table 5. Comparing the performance of the models

\begin{tabular}{clcccc}
\hline Parameter & Method & MAE & RMSE & MAPE & $\mathrm{R}^{2}$ \\
\hline \multirow{4}{*}{ SFC } & ELM & 28.65036 & 42.47032 & 5.585456 & 0.938683 \\
& BP & 16.7275 & 21.53959 & 3.239972 & 0.984228 \\
& RBF & 20.70679 & 32.52362 & 3.670709 & 0.964041 \\
\hline \multirow{4}{*}{ TE } & ELM & 0.826071 & 1.160392 & 4.718433 & 0.928886 \\
& BP & 0.818571 & 1.18921 & 4.339911 & 0.92531 \\
& RBF & 2.055 & 2.73098 & 13.24645 & 0.606102 \\
\hline \multirow{4}{*}{ EGT } & ELM & 6.659286 & 8.168776 & 3.572118 & 0.969049 \\
& BP & 13.72929 & 17.26228 & 7.287562 & 0.861783 \\
& RBF & 12.99821 & 15.46812 & 7.093514 & 0.889021 \\
\hline \multirow{4}{*}{ HC } & ELM & 2.710714 & 3.530919 & 4.570164 & 0.987182 \\
& BP & 3.589643 & 5.062206 & 5.252892 & 0.973654 \\
& RBF & 3.973929 & 4.922028 & 6.045235 & 0.975093 \\
\hline \multirow{3}{*}{ NO $_{x}$} & ELM & 40.91071 & 51.90364 & 6.082621 & 0.950288 \\
& BP & 68.29607 & 99.22855 & 11.69917 & 0.818308 \\
& RBF & 109.2182 & 139.4934 & 19.12019 & 0.640937 \\
\hline \multirow{2}{*}{ SO } & ELM & 0.596679 & 0.933658 & 3.958771 & 0.996605 \\
& BP & 0.863214 & 1.324569 & 7.240231 & 0.993167 \\
& RBF & 1.363857 & 2.155505 & 10.73633 & 0.981905 \\
\hline
\end{tabular}

Generally, when it is obtained the smaller the MAE, RMSE and MAPE, and the higher $\mathrm{R}^{2}$, then the model accuracy is the better. In addition, according to the error results obtained, the most suitable models for the investigated parameters are given in Table 6, and computational time for each model is given in Table 7. Tables show that ELM performed better than the traditional ANN (i.e., BP and $\mathrm{RBF}$ ) in terms of estimation accuracy (for emissions and EGT) and computational time (for all). The overall error results showed that MAPE, RMSE and MAPE errors of ELM, which are 13.39, 18.03 and 4.75 , respectively, is smaller than those of both BP and RBF (they are 17.34, 24.27 and 6.51 for BP and 25.05, 32.88 and 9.99 for RBF, respectively). Furthermore, $\mathrm{R}^{2}$ of ELM is 0.96 , and it is higher than $R^{2}$ values of both BP and RBF (0.93 and 0.84, respectively). ELMs' training time is 15 and 30 times faster than that of $\mathrm{BP}$ and RBF. BP has given some successful results in estimating performance parameter, e.g. BSFC was estimated with highest accuracy by BP. The accuracy of the RBF is also acceptable in estimating $\mathrm{HC}$ and $\mathrm{SO}$ and it has a high test speed in comparison with BP while its accuracy is less than that of others because the RBF network requires good coverage of the input space by radial basis functions. The accuracy of BP depends on tuning all network parameters (weights and biases) until the error does not decrease anymore [12] therefore BP can easily fall into local minima when there is a much deeper global minimum [21, 25]. Mao et al. [33] demonstrated that ELM tends to get unsatisfactory results when facing non-linear datasets. As seen in Table 7, ELM is much faster 
than $\mathrm{BP}$ and RBN. The BP, gradient-based learning, has very slow training stage because it needs much iteration for optimizing the network parameters. The long training process may be the result of non-optimum learning rate and momentum [21]. The results obtained in this study is agreed with the literature view, as it was reported by Huang et al. [21] that the learning speed of ELM can be thousands of times faster than BP. The results show that ELM can be easily used with high accuracy because (1) it is not only tends to reach the smallest training error but also the smallest norm of weights that means the network tends to have better generalization performance [21], (2) it is simple and needs less parameter (transfer function and number of neurons in hidden layer) than traditional classic gradient-based learning algorithms (number of hidden layer, number of neurons in hidden layer, transfer function, learning rate, momentum, maximum number of iteration), (3) optimization of ELM weights in hidden layer are calculated analytically and input weights and biases are determined randomly. Therefore, there is no any trouble of ELM such as over or under trained and local minima.

Table 6 . The most suitable method for each parameter

\begin{tabular}{ll}
\hline Parameter & Method \\
\hline SFC & BP \\
Efficiency & ELM, BP \\
EGT & ELM \\
HC & ELM, BP, RBF \\
NOx & ELM \\
SO & ELM, BP, RBF \\
\hline
\end{tabular}

Table 7. Computational time for each model (s)

\begin{tabular}{llcccccc}
\hline Method & Stage & SFC & TE & EGT & $\mathrm{HC}$ & $\mathrm{NO}_{\mathrm{x}}$ & $\mathrm{SO}$ \\
\hline \multirow{2}{*}{ ELM } & Train & 0.0468 & 0.0312 & 0.0312 & 0.0312 & 0.0312 & 0.0468 \\
& Test & 0.0312 & 0 & 0 & 0 & 0 & 0 \\
\hline \multirow{2}{*}{ BP } & Train & 0.7800 & 1.0296 & 0.9516 & 0.8268 & 1.6224 & 0.9516 \\
& Test & 0.0312 & 0.0312 & 0.1248 & 0.1404 & 0.1248 & 0.1872 \\
\hline \multirow{2}{*}{ RBF } & Train & 0.6084 & 0.5304 & 0.5304 & 0.5616 & 0.4992 & 0.5460 \\
& Test & 0 & 0.0156 & 0 & 0 & 0 & 0 \\
\hline
\end{tabular}

\section{Conclusion}

In this study, ELM, BP and RBF models have been successfully constructed to estimate the emission parameters of a biodiesel-fueled generator-set. The accuracy of estimations could be improved by employing ELM despite the number of dataset for training is small. A comparison of actual and estimated values showed that the correlation of the results agrees well with each other. Also, the comparison between ELM and traditional ANN techniques revealed that ELM performed better than BP and RBF, and the slower train stage is observed with BP in terms of MAE, MAPE, RMSE and $\mathrm{R}^{2}$ which are resulted from high generalization capacity and simpler optimization. Also, the lower accomplishment of BP may be caused by not having enough number of train data, local minima problem or complexity of determining the BP parameters. As a result, this work contributes to existing knowledge by providing the applicability of ELM which is a newly employed model in this area.

\section{Acknowledgements}

This work was supported by the Unit of Scientific Research Projects of Batman University, Turkey with Research Project No. BAP-2010-MF-01.

\section{References}

[1] C. Sayin, M. Canakci, Effects of injection timing on the engine performance and exhaust emissions of a dual-fuel diesel engine, Energ. Convers. Manage. 50(1) (2009) 203-13. 
[2] A. Mucak, M. Karabektas, C. Hasimoglu, G. Ergen, Performance and Emission Characteristics of a Diesel Engine Fuelled with Emulsified Biodiesel-Diesel Fuel Blends. International Journal of Automotive Engineering and Technologies, 5(4), (2016), 176-185.

[3] C. Haşimoğlu, M. Ciniviz, İ. Özsert, Y. İçingür, A. Parlak, M. S. Salman, Performance characteristics of a low heat rejection diesel engine operating with biodiesel. Renewable energy, 33(7), (2008) 1709-1715.

[4] M.S. Graboski, R.L. McCormick, Combustion of fat and vegetable oil derived fuels in diesel engines, Progress Energy Combust Sci. 24(2) (1998) 125-64.

[5] EC European Commission, Directive 2009/28/EC of the European Parliament and of the Council of 23 April 2009 on the promotion of the use of energy from renewable sources and amending and subsequently repealing Directives 2001/77/EC and 2003/30, Official Journal of the European Union Belgium (2009).

[6] Venkata Ramanan, M., Yuvarajan, D., Emission analysis on the influence of magnetite nanofluid on methylester in diesel engine, Atmospheric Pollution Research (2015), http://dx.doi.org/10.1016/j.apr.2015.12.001

[7] Ş. Altun, C. Öner, Gaseous emission comparison of a compression-ignition engine fueled with different biodiesels, International J Environ. Sci. Techn. 10(2) (2013) 371-6.

[8] M. Lapuerta, J.M. Herreros, L.L. Lyons, R. García-Contreras, Y. Briceño, Effect of the alcohol type used in the production of waste cooking oil biodiesel on diesel performance and emissions, Fuel 87(15) (2008) 3161-9.

[9] B. Kegl, NOx and Particulate Matter (PM) Emissions Reduction Potential by Biodiesel Usage, Energy Fuels 21 (2007) 3310-16.

[10] O.S. Valente, V.M.D. Pasa, C.R.P. Belchior, J.R. Sodré, Exhaust emissions from a diesel power generator fuelled by waste cooking oil biodiesel, Sci Total Environ. 431 (2012) 57-61.

[11] Ş. Altun, Effect of the degree of unsaturation of biodiesel fuels on the exhaust emissions of a diesel power generator, Fuel 117 (2014) 450-7.

[12] E. Alpaydin, Introduction to machine learning, $2^{\text {nd }} E d$. MIT press (2010) 273-318.

[13] W. S. McCulloch, W. Pitts, A logical calculus of the ideas immanent in nervous activity, The bulletin of mathematical biophysics, 5(4) (1943) 115-33.

[14] W.K. Yap, V. Karri, Comparative analysis of artificial neural networks and dynamic models as virtual sensors, Appl. Soft Computing 13(1) (2013) 181-8.

[15] G. Kokkulunk, E. Akdogan, V. Ayhan, Prediction of emissions and exhaust temperature for direct injection diesel engine with emulsified fuel using ANN, Turkish J Electrical Engineering and Computer Sciences 21 (2013) 2141-52.

[16] R.S. Kumar, R. Manimaran, V. Gopalakrishnan, Performance and Emission Analysis Using Pongamia Oil Biodiesel Fuel with an Artificial Neural Network, Advanced Engineering and Applied Sciences: An International Journal 3(1) (2013) 17-20.

[17] K.B. Patel, T.M. Patel, Performance Modeling of Single Cylinder Diesel Engine for Pyrolysis Oil and Diesel Blend using Neural Networks, International Journal of Emerging Technology and Advanced Engineering 3(1) (2013) 193-5.

[18] S. Kumar, P. S. Pai, B. R. Rao, Radialbasis-function-network-based prediction of performance and emission characteristics in a bio diesel engine run on WCO ester, Advances in Artificial Intelligence 10 (2012) 1-7.

[19] W. Shi, J. Yang, T. Tang, RBF NN based marine diesel engine generator modeling, in: American Control Conference, 2005, pp. 2745-9.

[20] G.B. Huang, Q.Y. Zhu, C.K. Siew, Extreme learning machine: a new learning scheme of feedforward neural networks, in: Proceedings of the 2004 International Joint Conference on Neural Networks, vol. 2, 2004, pp. 985-90.

[21] G. B. Huang, Q. Y. Zhu, C. K. Siew, Extreme learning machine: theory and applications, Neurocomputing 70(1) (2006) 489-501. 
[22] K. I. Wong, P. K. Wong, C. S. Cheung, C. M. Vong, Modeling and optimization of biodiesel engine performance using advanced machine learning methods, Energy 55 (2013) 519-28.

[23] K. I. Wong, P. K. Wong, C. S. Cheung, C. M. Vong, Modelling of diesel engine performance using advanced machine learning methods under scarce and exponential data set, Applied Soft Computing 13(11) (2013) 4428-41.

[24] Ş. Altun, F. Yasar, A comparison of performance and emissions of a diesel power generator fueled with biodiesels from waste frying oils, in: Proceedings of EuroTecS-2013, vol. 1, 2013, pp.139-143. Sarajevo, Bosnia and Herzegovina.

[25] S. Tasdemir, I. Saritas, M. Ciniviz, N. Allahverdi, Artificial neural network and fuzzy expert system comparison for prediction of performance and emission parameters on a gasoline engine. Expert Systems with Applications, 38(11), (2011), 13912-13923.

[26] İ. Örs, V. Bakırcioğlu, An Experimental and ANNs Study of the Effects of Safflower Oil Biodiesel on Engine Performance and Exhaust Emissions in a CI Engine. International Journal of Automotive Engineering and Technologies, 5(3), (2016), 125-135.

[27] ÖF. Ertuğrul, Y. Kaya, A detailed analysis on extreme learning machine and novel approaches based on ELM, American Journal of Computer Science and Engineering 1(5), (2014) 43-50.,

[28] ÖF. Ertuğrul, Ş. Altun, Developing Correlations by Extreme Learning Machine for Calculating Higher Heating Values of Waste Frying Oils from their Physical Properties, Neural Computing and Applications, (2016), DOI: 10.1007/s00521-016-2233-8.

[29] D.E. Rumelhat, G.E. Hinon, R.J. Williams, Learning representations by backpropagating errors, Nature 323 (1986) 5336.

[30] D. S. Broomhead, D. Lowe, Radial basis functions, multi-variable functional interpolation and adaptive networks (No.RSRE-MEMO-4148), Royal Signals and Radar Establishment Malvern, UK (1988).

[31] G. B. Huang, H. A. Babri, Upper bounds on the number of hidden neurons in feedforward networks with arbitrary bounded nonlinear activation functions, Neural Networks 9(1) (1998) 224-229.

[32] R. Kohavi, A study of cross-validation and bootstrap for accuracy estimation and model selection, IJCAI 14(2) (1995) 11371145.

[33] W. Mao, Y. Wang, X. Cao, Y. Zheng, Mixture Regression Estimation based on Extreme Learning Machine, Journal of Computers, 8(11) (2013) 2925-2933. 